\title{
The Influence of Geomagnetic Activity on the Radial Variation of the Magnetospheric Electric Field Between $L=4$ and 10
}

\author{
STANLEY M. KAYE' \\ Space Science Laboratory, Lockheed Palo Alto Research Laboratory, Palo Alto, California 94304 \\ MARGARET G. KIVELSON \\ Department of Earth and Space Sciences and Institute of Geophysics and Planetary Physics, University of California, Los Angeles \\ Los Angeles, California 90024

\begin{abstract}
In this report we use electric field data measured aboard the low-altitude Ogo 6 spacecraft [Heppner, 1972] to determine the gross radial variation of the equatorial convection electric field. To transform the electric field from low altitudes to the equatorial magnetosphere, we assume that the earth's magnetic field is dipolar and that $E \cdot B=0$. To determine the radial variation of the electric field, we fit the data between $L=4$ and 10 to a power law function of the form $\Phi=c L^{\kappa} \sin \phi$ where $\mathbf{E}=-\nabla \Phi$ and $\phi$ is azimuth. We find that in general the convection electric field magnitude decreases with decreasing radial distance $(\kappa>1)$, implying that the field is partially shielded from the inner magnetosphere. This result is consistent with prior theoretical and experimental work. We find, in addition, that the magnitude of the radial variation of the electric field is a function of geomagnetic activity such that $\boldsymbol{\kappa}$ varies inversely with $K p$ for $K p \leq 3$, and $k \simeq 1$ for $K p \geq 3$. We argue that this $k-K p$ relation probably reflects the fact that the electric field is shielded only within the ring current which moves radially inward as $K p$ increases. Only for $K p \leq 3$ does the region of measurement $(L=4$ to 10$)$ include the region of shielded electric field in and near the ring current inner edge. For $K p \geqslant 3$, the measurements sample the electric field only outside the region of shielding.
\end{abstract}

\section{INTRODUCTION}

There has recently been renewed interest in using the concept of particle convection in the earth's magnetosphere to explain both steady state and time-dependent particle observations. Two different, but not necessarily conflicting, approaches to the problem have been taken. In theoretical studies based on the self-consistency argument of Vasyliunas [1970], Jaggi and Wolf [1973] and Wolf [1974] numerically calculated the time evolution of the convection electric field following a sudden enhancement of its magnitude. They realized that the field magnitude was partially influenced by the distribution of particles in the magnetosphere, and their calculation showed how the spatial particle distribution, modified by the convection electric field, would in tum modify the electric field. Their results predicted extremely strong shielding of the electric field earthward of the ring current inner edge. Using a fluid approach to the same problem, Southwood [1977] arrived at similar conclusions.

The shielding results from the closure of magnetospheric currents in the resistive ionosphere. In response to an increase in the convection electric field magnitude, particles convect earthward and set up pressure gradients which, through momentum conservation, give rise to currents perpendicular to the magnetic field. The perpendicular current is not divergence free if there is any component which flows across contours of constant flux tube volume, and consequently a net field-aligned current is produced [Southwood, 1977]. In order for the total current density to be divergence free, these fieldaligned currents close in the resistive ionosphere. The resulting ionospheric potential drop maps back into the magnetosphere and reduces the initial convection field.

\footnotetext{
' Now at Plasma Physics Laboratory, Princeton University, Princeton, New Jersey 08540.
}

Copyright (C) 1981 by the American Geophysical Union.
Although the approach to modeling the electric field that was taken by Jaggi and Wolf [1973] and Wolf [1974] was a self-consistent and consequently physically valid one, the need to assume a magnetospheric particle distribution and repeat the iterative procedure performed by these authors is both time consuming and cumbersome. In addition, the results of the computation are extremely sensitive to the choice of a distribution, and the particle population in the magnetosphere is not well known. An alternative approach for performing convection calculations, then, is to assume a form for the electric field with large-scale properties consistent with the Jaggi and Wolf [1973], Wolf [1974], and Southwood [1977] results. Such a form was proposed by Volland [1973], who assumed that the total electric field in the equatorial plane of the inner magnetosphere $(L<15)$ could be derived from the electrostatic potential given by

$$
\Phi(\mathrm{kV})=c L^{\kappa} \sin \phi+\frac{91 \mathrm{kV}}{L}
$$

where $c$ is proportional to the cross-magnetospheric potential drop in kilovolts, $L$ is equatorial distance in earth radii, $\phi$ is azimuth measured counterclockwise from noon, $91 \mathrm{kV}$ is the corotation potential, and $\boldsymbol{\kappa}$ is a parameter which characterizes the extent to which the electric field penetrates to low $L(\kappa=1$ for a uniform field, and it increases with increasing shielding). Implicit in the form of (1) are the assumptions that the earth's magnetic field is dipolar, $\partial \mathbf{B} / \partial t=0$, and $\mathbf{E} \cdot \mathbf{B}=0$. The electrostatic potential given in (1) has been the basis for convection studies by Kavanagh et al. [1968], Chen [1970], Stern [1975], Kivelson and Southwood [1975], Cowley and AshourAbdalla [1976a, b], Ejiri [1978], Ejiri et al. [1978], Maeda et al. [1978], Kaye and Kivelson [1979], and Southwood and Kaye [1979].

Various studies have been made to determine the value of $\kappa$; the self-consistent work performed by Jaggi and Wolf 
[1973], Wolf [1974], and Southwood [1977] suggests that $\kappa>1$. Volland [1973] and Ejïi et al. [1978] used plasmapause elongation measurements, and Volland [1973] further used observations of ionospheric electric fields, equivalent currents, and field-aligned currents to demonstrate that $\kappa \simeq 2$. In a recent paper, Southwood and Kaye [1979] developed some formulae to approximate static convection boundaries and used these formulae to determine $\kappa$ from several different observations. They found that either $\kappa=1$ or $\kappa=2$ fit the measurements better than larger values of $\kappa$.

A shortcoming of these previous studies is the treatment of $\boldsymbol{x}$ as a parameter independent of the level of geomagnetic activity. It must be recognized that the magnitude of the electric field, and therefore the amount of ring current particle penetration, depends on $K p$ [Kivelson, 1976]. Since it is these ring current particles that are responsible for the shielding, we cannot ignore the possibility that $\kappa$ depends on $K p$. In the following analysis we too set about to determine the value of $\kappa$, but we let $K p$ be an independent parameter. We find that $x$ depends on $K p$ in a manner consistent with expectations based on convection theory.

\section{ANALYSIS}

Our approach to determining $\boldsymbol{\alpha}$ is to analyze the radial dependence of directly measured large-scale electric field magnitudes. Such a data set is available from the dc electric probe measurements aboard the low-altitude, polar-orbiting Ogo 6 satellite [Heppner, 1972]. The Ogo 6 probe operated for approximately 2 weeks, from June 9 to 22, 1969. During this time the satellite crossed the polar cap approximately in the dawn-dusk meridian with an apogee of $1100 \mathrm{~km}$ and a perigee of $400 \mathrm{~km}$. The data presented by Heppner [1972] were reduced so that the corotation electric field and the electric field due to the satellite's motion through the earth's magnetic field were removed. The remaining (convection) electric field was in the north-south (latitudinal) direction at the low-altitude points of observation.

To determine $\kappa$, we must first transform the electric field magnitude and position of observation to corresponding values in the equatorial plane. We first assume that the earth's magnetic field is that of a dipole so that

$$
L \cos ^{2} \Lambda=1
$$

where $\Lambda$ is invariant latitude, which we take to be $\lambda$, the latitude of observation. We next assume that there are no potential drops along the field line, so that at dawn or dusk the total potential drop across a given increment of geomagnetic latitude at low altitude

$$
\Delta \Phi=E_{i} R_{E} \Delta \lambda
$$

is equal to the potential drop across a conjugate region at the equator

$$
\Delta \Phi=E_{m} R_{E} \Delta L
$$

Consequently, the low-altitude electric field $E_{i}$ is related to $E_{m}$, the electric field in the equatorial plane, by

$$
E_{m}=E_{i} \frac{\Delta \lambda}{\Delta L}
$$

where by (2)

$$
\frac{d \lambda}{d L}=\frac{\cot \lambda}{2 L}
$$

Equations (2), (5), and (6) allow us to transform both the position of observation and electric field magnitudes from low altitudes to their values at the geomagnetic equator under the assumptions of our model.

We chose to analyze time periods during which the electric field exhibited a smooth variation with latitude, since the aim of the study is to determine the gross variation of field magnitude with $L$. Periods during which the field magnitude varied rapidly, due to either temporal effects or spatial fine structure, were ignored. From (1) it is seen that the relation between $\left|\mathbf{E}_{m}\right|$ and $L$ is given by

$$
\left|\mathbf{E}_{m}\right| \propto L^{\kappa-1}
$$

so that $\kappa$ may be determined directly from the slope of $\log \left|E_{m}\right|$ versus $\log L$.

A linear least squares fit to the data $\left(\log \left|\mathbf{E}_{m}\right|\right.$ versus $\left.\log L\right)$ was performed to calculate the slope, $\kappa-1$, and $y$ intercept. To suppress the effect of spurious variations of the field magnitude, all points which were outside one standard deviation of the calculated least squares fit were removed, and a linear least squares fit to the remaining points was performed.

The values of $\kappa$ calculated in this manner are given in Table 1. Given in the table are the dates and times of the polar cap passes used in this analysis, as well as whether the pass was over the northern or southern polar cap during the morning or evening hours. Also given in the table is the average magnitude of the measured polar cap electric field, the width of the polar cap (also determined from the measurement), the corresponding cross-polar cap potential drop, and $K p$ during the time of observation. Within the limited number of passes available for the analysis, there appears to be no clear correlation between $\kappa$ and $\left|E_{p c}\right|, \Delta \lambda_{p c}$ or $\Phi_{p c}$. However, the relation between $\kappa$ and $K p$ which emerges in Table 1 is shown graphically in Figure 1, which illustrates two points. First, most of the passes exhibit $1 \leq \kappa \leq 2$, which is consistent with the results of Volland [1973], Ejiri et al. [1978], and Southwood and Kaye [1979]. Second, and not illustrated in any of the previous work, is the clear tendency for $\kappa$ to decrease as $K p$ increases.

Both these results also emerge in the work of Rycroft and Thomas [1970]. They used the Carpenter [1966] whistler observations to determine an empirical relation between the plasmapause position as a function of local time and $K p$. Using a method similar to that used by Ejiri et al. [1978], we found that the Rycroft and Thomas [1970] empirical relation produced $1 \leq \kappa \leq 2$ for all levels of geomagnetic activity, with a tendency for $\kappa$ to decrease as $K p$ increases. This latter result will be discussed later.

In Figure 1 we see that there are two intervals, when $K p=$ 3 , for which the values of $\kappa$ are anomalous, with $\kappa=7.3$ and $\kappa$ $=-0.2$. The first interval, which occurred on June 13, 1969, during a morning pass over the northern polar cap, suggests a field that was practically completely shielded from the inner magnetosphere, and the second interval, which occurred on June 13, 1969, during an evening pass over the southern polar cap, depicts a field which increased in magnitude as $L$ decreased. To examine these periods in more detail, we plot $\log$ $\left|E_{m}\right|$ versus $\log L$ in Figures 2 and 3 for the two intervals. To compare these anomalous periods with a more typical one, we have plotted in Figure 4 an interval on June 11, 1969, during a morning pass over the northern polar cap. During this interval, $K p=3-$ and $\kappa=1.6$.

The interval during which $\kappa=7.3$ is shown in Figure 2, and the very steep gradient of field magnitude with $L$ is evident, 
TABLE 1. Ogo 6 Passes Used for Calculating $\kappa$

\begin{tabular}{|c|c|c|c|c|c|c|c|c|}
\hline Date & $\begin{array}{c}\text { Time, } \\
\text { UT }\end{array}$ & PC & $\mathbf{E} / \mathbf{M}$ & $\boldsymbol{\kappa}$ & $\begin{array}{c}E_{p c} \\
\mathrm{mV} / \mathbf{m}\end{array}$ & $\frac{\Delta \lambda_{p o}}{\operatorname{deg}}$ & $\begin{array}{l}\Phi_{p c} \\
\mathbf{k V}\end{array}$ & $K p$ \\
\hline \multirow[t]{2}{*}{ June 11, 1969} & 0504 & $\mathbf{N}$ & $\mathbf{E}$ & 2.1 & 25 & 27 & 75 & $2-$ \\
\hline & & & $\mathbf{M}$ & 2.4 & 25 & 27 & 75 & $2-$ \\
\hline \multirow[t]{2}{*}{ June 11, 1969} & 0820 & $\mathbf{N}$ & $\mathbf{E}$ & 2.1 & 20 & 32 & 72 & 3- \\
\hline & & & $\mathbf{M}$ & 1.6 & 20 & 32 & 72 & 3- \\
\hline \multirow[t]{5}{*}{ June 13, 1969} & 0150 & $\mathbf{N}$ & $\mathbf{E}$ & 1.5 & 25 & 34 & 94 & 3 \\
\hline & & & $\mathbf{M}$ & 7.3 & 25 & 34 & 94 & 3 \\
\hline & 0236 & $\mathbf{S}$ & $\mathbf{M}$ & 1.0 & 10 & 36 & 40 & 3 \\
\hline & & & $\mathbf{E}$ & -0.2 & 10 & 36 & 40 & 3 \\
\hline & 0420 & $\mathbf{S}$ & $\mathbf{M}$ & 0.8 & 20 & 32 & 71 & 4 \\
\hline \multirow[t]{2}{*}{ June 14, 1969} & 0747 & $\mathbf{N}$ & $\mathbf{E}$ & 0.7 & 26 & 33 & 94 & 5 \\
\hline & & & $\mathbf{M}$ & 1.4 & 26 & 33 & 94 & 5 \\
\hline \multirow[t]{2}{*}{ June 16, 1969} & 0118 & $\mathbf{N}$ & $\mathbf{E}$ & 1.1 & 30 & 33 & 110 & 3- \\
\hline & & & $\mathbf{M}$ & 1.0 & 30 & 33 & 100 & 3- \\
\hline \multirow[t]{2}{*}{ June 22, 1969} & 0153 & $\mathbf{N}$ & $\mathbf{E}$ & 3.1 & 12 & 27 & 31 & 0 \\
\hline & & & $\mathbf{M}$ & 5.3 & 12 & 27 & 31 & $\mathbf{0}$ \\
\hline
\end{tabular}

Date and time of the pass are given, also whether the pass was over the southern (S) or northern $(\mathrm{N})$ polar cap (PC) during the morning (M) or evening (E). Also given are the calculated values of $k$, average electric field across the polar cap $\left(E_{p c}\right)$, width of the polar cap $\left(\Delta \mathrm{\Lambda}_{p c}\right)$, the corresponding potential drop across the polar cap $\left(\Phi_{p c}\right)$, and $K p$ during the pass.

but there is no apparent spatial or temporal irregularity which would account for such large $\kappa$. Aside from the variation in $|\mathbf{E}|$ near $L=8$ where the assumption $E_{\|}=0$ may be invalid, the field was a smoothly varying function of $L$. We do not believe that this large value of $\kappa$ resulted from strong temporal changes in the convection electric field strength, for $K p$ varied only between 2 and 4 for two days prior to the interval shown in Figure 2. Therefore it appears that the shielding during this time period was virtually complete and that the large value of $\kappa$ represented a true spatial variation of the electric field magnitude.

A large nonmonotonic variation of the electric field magnitude with $L$ was seen during the interval for which $x$ was found to be -0.2 . which is shown in Figure 3. The field magnitude was significantly reduced at $L$ values between 6 and 9 , and it is clear that this large variation invalidates the linear regression technique used to determine $\kappa$. For values of $L<6$, the field varied with $L$ at a rate corresponding to $\kappa \simeq 2$, but it is not known whether spatial or temporal variations in $E$ were responsible for the low magnitude at large $L$. We do note that the region of low field magnitude corresponded to the auroral zone where parallel fields are known to exist [Mozer et al., 1977]. Therefore the $\kappa=-0.2$ result is one which does not satisfy the assumptions of our analysis and should not be regarded as meaningful.

\section{Discussion}

The results of the analysis presented in this study fit very nicely with previous determinations of $\kappa$, with $\kappa=1$ to 2 for most cases. It seems that $\kappa$ varies rather weakly with $K p$, although rather large values of $k$ are found for $K p=0$ (see Figure 1). There is a clear tendency for $k$ to decrease with increasing geomagnetic activity.

The observed $\kappa-K p$ dependence can be interpreted on the basis of several features of the shielding mechanism proposed by Jaggi and Wolf [1973] and Southwood [1977]. One pertinent feature is the time scale of the shielding process. The shielding time scales estimated by Jaggi and Wolf [1973] and Wolf [1974] were of the order of hours on the dayside, but because of the relatively low ionospheric conductivity at night the estimated shielding time scale there was of the ordér of minutes. However, Southwood [1977] pointed out that nightside shielding would not be complete until the dayside shielding was, so that even for the nightside the shielding time scale should be of the order of hours. We can infer from the monotonic relation between $\kappa$ and $K p$ in Figure 1 that the time scale for changes in $\kappa$ is comparable to the $K p$ interval of 3 hours. If the time scale for changes in $k$ were not of the order of the 3hour $K p$ interval, we would not expect such a systematic relation of $\kappa$ and $K p$, but rather one in which $\kappa$ is extremely variable within a single $K p$ interval.

Another feature of the model is the nonuniform nature of the shielded field and the relation of the shielding to the position of the inner edge of the ring current. The results of Jaggi and Wolf [1973], Wolf [1974], and Southwood [1977] indicate that the convection electric field is most effectively shielded in

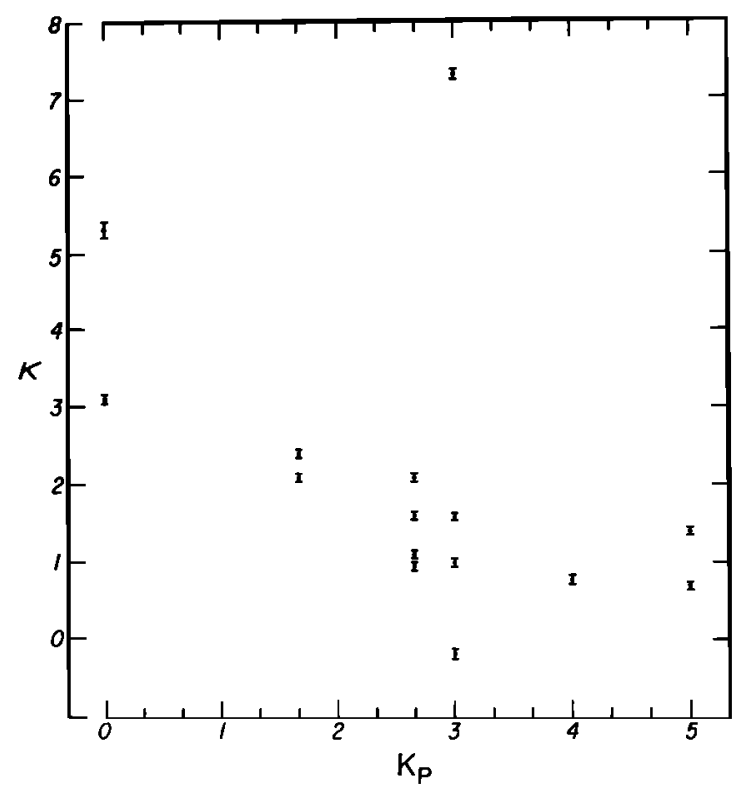

Fig. 1. $x$ as a function of $K p$ calculated from the Ogo 6 electric field measurements for the passes given in Table 1 . The error bars on each point represent the one standard deviation range for the calculated fit. 


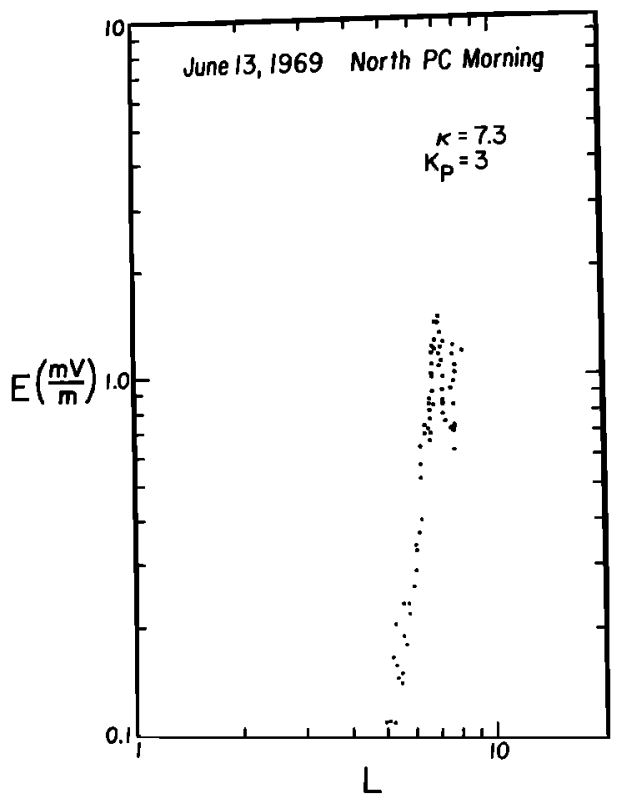

Fig. 2. The magnetospheric electric field magnitude is plotted as a function of $L$ for the Ogo 6 pass on June 13, 1969, over the morning northern polar cap. The method for determining $E$ and $L$ is given in the text.

the region earthward of the ring current inner edge. Consequently, the magnitude of the shielding determined from the measured field must depend on whether the electric field is measured earthward or antiearthward of the ring current inner edge.

Not only the position of the ring current inner edge but also its spatial structure influences the electric field shielding. In the work of Jaggi and Wolf [1973] and Southwood [1977] the ring current inner edge was assumed to be a sharp boundary for analytical convenience. However, the observations of Smith and Hoffman [1974] and Ejiri [1978] indicate that the

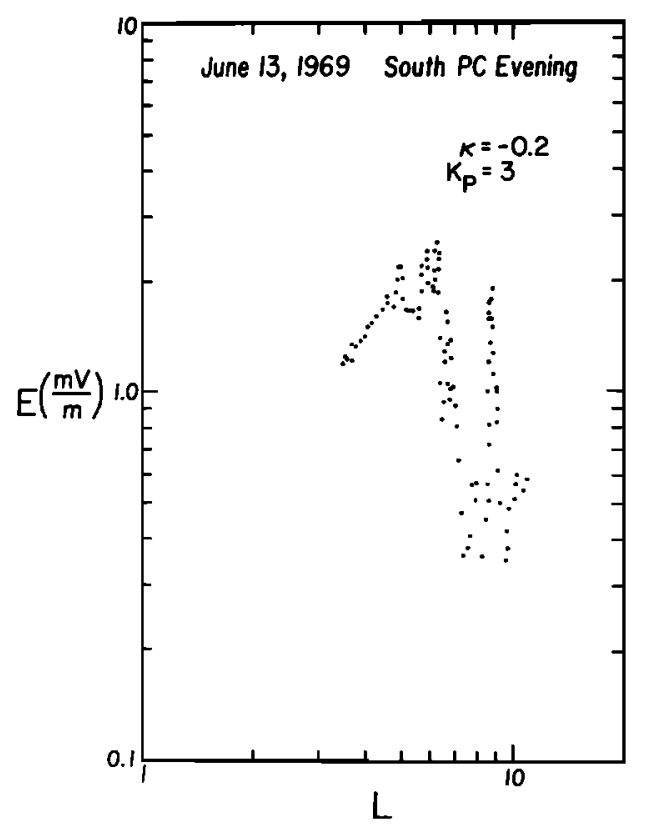

Fig. 3. Same as Figure 2 for June 13, 1969, over the evening southern polar cap.

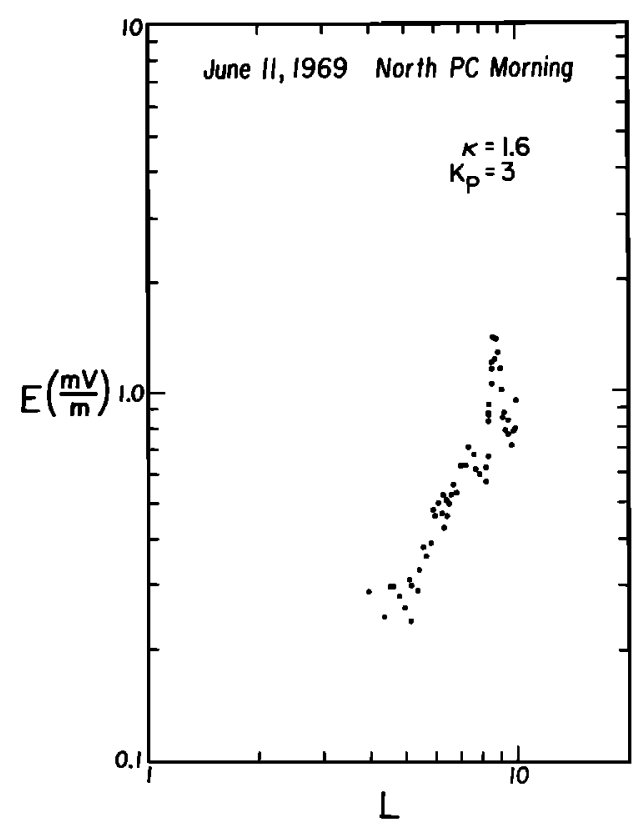

Fig. 4. Same as Figure 2 for June 11, 1969, over the morning northern polar cap.

ring current inner edge can be broad, with thicknesses of at least $1 \boldsymbol{R}_{E}$. The inner edge is a difficult feature to define quantitatively even in theory, since the positions of particle convection boundaries are themselves energy dependent [Kivelson and Southwood, 1975; Southwood and Kaye, 1979]. To date there has been no work on the magnitude of shielding within such a diffuse boundary, although intuition tells us that the transition from a shielded to an unshielded region of space (or vice versa) would be gradual through a diffuse boundary rather than sudden. Consequently, we envision a situation in which the electric field magnitude is large and constant with radial distance beyond the ring current inner edge $(\kappa=1)$, where the electric field varies strongly with radial distance through a transition region that is the ring current inner edge $(1<\kappa \leq 2)$, and where the electric field is shielded and small in the region earthward of the ring current inner edge ( $k \geq 2)$.

With these ideas in mind, we now propose a possible way in which the observed $\boldsymbol{k}-\boldsymbol{K} p$ relation could be explained. Here we examine the spatial relation between the ring current inner edge and the region within which the electric field was measured. It is the magnitude of the cross-magnetospheric potential drop (and thus the convection electric field) that controls the distance to which ring current particles can penetrate, and the magnitude of the potential drop is proportional to $K p[K i$ velson, 1976]. For $L=4$ to 10, the region of the Heppner [1972] observations, we would expect to see a field that is strongly shielded when the ring current inner edge is at large $L$ (outside the region of measurement); this occurs for small electric field values $(K p \leq 3)$. Little shielding ( $k$ small) is expected when the region of measurement is at higher $L$ than the ring current inner edge, and this situation occurs when the electric field is large $(K p \geq 3)$.

The above scenario qualitatively fits the observed $k-K p$ dependence as well as the considerations discussed above. We first note that convection time scales, the time required for particles to come to semi-equilibrium following a change in the convection electric field strength, is several hours [Ejiri, 
1978], roughly equivalent to the time scale for changes in $\kappa$ as discussed at the beginning of this section. Thus changes in $k$ that we observe keep step with radial changes in ring current particle convection boundaries, consistent with the idea that the electric field varies spatially within the ring current particle distribution and that the position of the ring current distribution depends on $K p$. That the variation of $\kappa$ with $K p$ is smooth suggests that the transition between shielded and unshielded spatial regions is gradual. We suggest here that such a gradual transition is further evidence for the diffuse nature of the ring current inner edge. We also note from Figure 1 that, excluding the $\kappa=-0.2$ interval, $\kappa \sim 1$ for most intervals in which $K p \geq 3$. This result suggests that for $K p \geq 3$ the ring current inner edge is situated close to the earth $(L \leq 4)$ and that we are sampling the region of space beyond the inner edge where we expect the electric field to be unshielded.

There is one additional comment that we should like to make at this point, and that is we might envisage a situation in which the causal relation between $\kappa$ and $K p$ is reversed, with $K \boldsymbol{p}$ depending on $\boldsymbol{k}$. Particles penetrate far earthward under the influence of an unshielded convection electric field (small к) and then precipitate owing to some loss mechanism such as pitch angle diffusion resulting from wave-particle interactions. This enhanced lower-latitude precipitation leads to an increase of geomagnetic activity as observed on the ground. A possible way of deciding on the correct causal relation between $\kappa$ and $K p$ is to examine the time delay between our calculated $\boldsymbol{\kappa}$ and some finer scale indicator of geomagnetic activity such as $A E$, and we are attempting such a correlation.

Acknowledgments. The authors wish to thank David Stern for useful suggestions concerning the inverse relation between $\boldsymbol{x}$ and $K p$. This research was supported by the Atmospheric Research Section of the National Science Foundation under grants ATM 74-23464 and ATM 79-23586, by the National Aeronautics and Space Administration under grant NGL 05-007-004, and by internal Lockheed independent research funds.

\section{REFERENCES}

Carpenter, D. L., Whistler studies of the plasmapause in the magnetosphere, temporal variations in the position of the knee, and some evidence of plasma motions near the knee, J. Geophys. Res., 71, 693, 1966.

Chen, A. J., Penetration of low-energy protons deep into the magnetosphere, J. Geophys. Res., 75, 2458, 1970.

Cowley, S. W. H., and M. Ashour-Abdalla, Adiabatic plasma convection in a dipole field: Electron forbidden-zone effects for a simple electric field model, Planet. Space Sci., 24, 805, 1976a.

Cowley, S. W. H., and M. Ashour-Abdalla, Adiabatic plasma con- vection in a dipole field: Proton forbidden-zone effects for a simple electric field model, Planet. Space Sci., 24, 821, $1976 b$.

Ejiri, M., Trajectory traces of charged particles in the magnetosphere, J. Geophys. Res., 83, 4798, 1978.

Ejiri, M., R. A. Hoffman, and P. H. Smith, The convection electric field model for the magnetosphere based on Explorer 45 observations, J. Geophys. Res., 83, 4811, 1978.

Heppner, J. P., Electric field variations during substorms: Ogo-6 measurements, Planet. Space Sci., 20, 1475, 1972.

Jaggi, R. K., and R. A. Wolf, Self-consistent calculation of the motion of a sheet of ions in the magnetosphere, J. Geophys. Res., 78, 2852, 1973.

Kavanagh, L., J. Freeman, and A. J. Chen, Plasma flow in the magnetosphere, J. Geophys. Res., 73, 5511, 1968.

Kaye, S. M., and M. G. Kivelson, Time-dependent convection electric fields and plasma injection, J. Geophys. Res., 84, 4183, 1979.

Kivelson, M. G., Magnetospheric electric fields and their variation with geomagnetic activity, Rev. Geophys. Space Phys., 14, 189, 1976.

Kivelson, M. G., and D. J. Southwood, Approximations for the study of drift boundaries in the magnetosphere, $J$. Geophys. Res., 80 , 3528, 1975.

Maeda, K., N. K. Bewtra, and P. H. Smith, Ring current electron trajectories associated with ELF emissions, J. Geophys. Res., 83, 4339, 1978.

Mozer, F. S., C. W. Carlson, M. K. Hudson, R. B. Torbert, B. Parady, J. Yatteau, and M. C. Kelley, Observations of paired electrostatic shocks in the polar magnetosphere, Phys. Rev. Lett., 38, 292, 1977.

Rycroft, M. J., and J. O. Thomas, The magnetospheric plasmapause and the electron density trough at the Alouette 1 orbit, Planet. Space Sci, 18, 65, 1970.

Smith, P. H., and R. A. Hoffman, Direct observation in the dusk hours of the characteristics of the storm time ring current particles during the beginning of magnetic storms, J. Geophys. Res., 79, 966, 1974.

Southwood, D. J., The role of hot plasma in magnetospheric convection, J. Geophys. Res., 82, 5512, 1977.

Southwood, D. J., and S. M. Kaye, Drift boundary approximations in simple magnetospheric convection models, J. Geophys. Res., 84, 5773, 1979.

Stern, D. P., The motion of a proton in the equatorial magnetosphere, J. Geophys. Res., 80, 595, 1975.

Vasyliunas, V. M., Mathematical models of magnetospheric convection and its coupling to the ionosphere, in Particles and Fields in the Magnetosphere, edited by B. McCormac, p. 60, D. Reidel, Hingham, Mass., 1970.

Volland, H., A semiempirical model of large-scale magnetospheric electric fields, J. Geophys. Res., 78, 171, 1973.

Wolf, R. A., Calculations of magnetospheric electric fields, in Magnetospheric Physics, edited by B. McCormac, p. 167, D. Reidel, Hingham, Mass., 1974.

(Received April 2, 1980; revised June 13, 1980; accepted July 8, 1980.) 